\title{
Allen J. Bard and Cynthia G. Zoski (Eds.): Electroanalytical Chemistry: A Series of Advances, Vol. 26
}

\author{
Grégoire Herzog ${ }^{1}$
}

(C) Springer-Verlag Berlin Heidelberg 2016

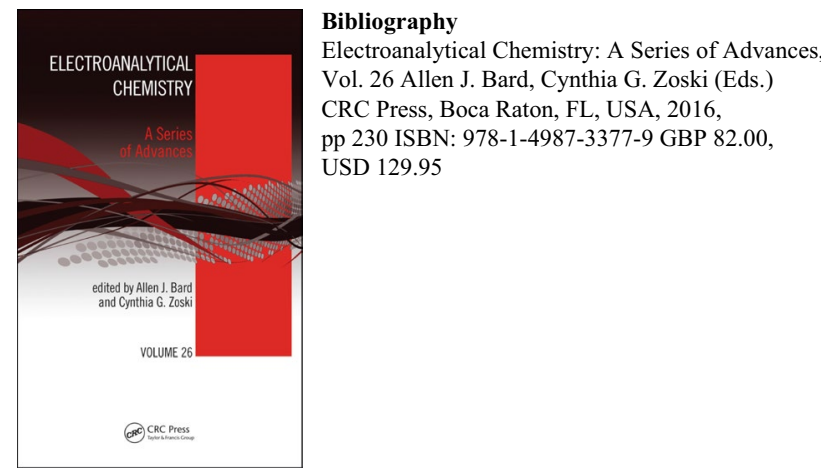

The latest volume of the collection Electroanalytical Chemistry has seen a revolution with the appearance of figures in colour. At last, if I dare say! It might seem incongruous, in 2016, to praise the use of colour as an innovation. Nevertheless, it is greatly appreciated as two of the three chapters are reporting on the latest advances of scanning electrochemical microscopy (SECM) and scanning ion conductance microscopy (SICM). The power of both these scanning techniques resides in their ability to provide images of surface reactivity at the nanoscale using an electrochemical probe in the form of a nano-pipette or a nano-electrode. Colour illustrations emphasize the potency of these techniques. The first chapter is devoted to the constant efforts of the SECM community to push forward the limits of the technique. Advances in the recent years are particularly focused on lowering two aspects: (1) the lateral resolution below $100 \mathrm{~nm}$, and (2) the nanogap between a tip and a substrate. The technological improvements (nanotip fabrication, its characterisation and its controlled positioning), which have enabled the latest advances, are detailed. The second chapter on SICM is written in the form of a tutorial review on the principle of the method and on the possibilities offered by the technique. The publication of these two chapters side-by-side is of great interest, since it allows the reader to have both techniques described in a single volume. The authors of both chapters highlighted in their text how SECM and SICM can be combined into a powerful tool offering the possibility of simultaneously measuring ion and faradaic currents.

I particularly enjoyed reading the third monograph on the modification of electrode surface via the grafting of diazonium salts. This chapter is very complete with over 450 references, and it constitutes a manual on the grafting of electrode surfaces. Both electrochemical and nonelectrochemical methods are detailed; the different types of surfaces on which a variety of diazonium salts can be grafted are classified. The mechanisms involved in the grafting processes and the characterisation of the surfaces modified are thoroughly explained. Such a chapter will be of great use to anyone wishing to modify a surface by the grafting methods.
Grégoire Herzog

gregoire.herzog@univ-lorraine.fr

1 CNRS-Université de Lorraine, Villers-lès-Nancy, France 